\title{
Assessment of Risk Factors For Patients With Thyroid Cancer in Mosul Teaching Hospitals
}

\author{
Dr. Taha Hassan Taha Alsaiq* Emad Elias Khaleel Albrimami **
}

\begin{abstract}
Background and Objectives: Thyroid cancer is the most common endocrine malignancy. Several risk factors were found to play a role in thyroid cancer. The aim of the study was to identify risk factors for thyroid cancer in Mosul city and to assess the relationship between some types of food and thyroid cancer.

Materials and Methods: The Non-experimental Quantitative design (a Case - Control approach) was used in this study. The study was conducted at AL-Jamhori Teaching Hospital, Al-Salam Teaching Hospital, and Nuclear Medicine and Oncology Hospital in Mosul city. The period from $18^{\text {th }}$. October, 2012 to $20^{\text {th }}$, August, 2013. A formal consent was taken from all participants (case group and control group) in questionnaire. A non probability purposive sample was selected that consisted (172) clients divided into two groups, a case group (86) clients with thyroid cancer and a control group (86) clients without goiter and thyroid cancer.

Results: According to the age, the highest percentage was in the age group (50-59 years),constituted (34\%)for the case group. The majority of the case group are female who were accounted $(78.0 \%)$ female to male ratio $4: 1$. The incidence of differentiated thyroid carcinoma according to histopathology was increased for women compared with men specially papillary thyroid carcinoma.

Conclusion: The statistical analysis shows that there are significant relationship in previous history for goiter, family history for thyroid cancer, and exposure to radio active iodine items with respect to the risk factors of thyroid cancer.
\end{abstract}

Keywords: Assessment, Risk Factors, Thyroid Cancer.

\section{INTRODUCTION}

Thyroid cancer represents a group of malignant tumors that affect the thyroid gland (NCI, 2012). Thyroid cancer including $91.2 \%$ of all endocrine new cancers and $56.5 \%$ of endocrine cancer deaths in 2002 in Iran. The difference between the total number of thyroidoriginating endocrine cancers and the ratio of total endocrine cancer deaths due to thyroid cancer are $91.2 \%$ and $56 / 5 \%$ respectively, showing a relatively weak and sluggish nature of most thyroid malignancies and their long-term survival (Harrison's, 2004).

Thyroid cancer is a relatively rare tumor, but is considered the most common tumor of the endocrine glands; it represents $1 \%$ of the overall cancer cases. The worldwide incidence ranges between 0.5 and 10 per 100,000 population (Marjani and Kabir, 2008).

Known risk factors for thyroid cancer include previous high dose environmental radioactive iodine exposure, previous childhood radiation to the head and neck, iodine deficiency, female gender, and family history of thyroid cancer (Sherman,2003). Latent period after child exposure is at least three to five years and in most cases occurs 20 to 40 years after the exposure (Boice, 2005

The principal clinical manifestation of thyroid cancer is a firm, fixed, small, rounded, painless mass or nodule that is felt during palpation of the gland. Only in rare instances are the symptoms of hyperthyroidism seen. (Barbra and Elaine, 2011). Thyroid nodules are common in adults and may be detected by palpation in $10 \%$ of women and $2 \%$ of men, the prevalence may be as high as $50 \%$ or more if sensitive imaging such as ultrasonography is used. The vast majority of thyroid nodules are benign and do not require urgent referral. Furthermore, thyroid cancer is uncommon in patients who are not euthyroid, and assessment of biochemical thyroid status is useful in deciding on the referral pathway by the general practitioner. (Hegedus, 2004).

The management of patients with thyroid cancer depends on the histological subtype and stage of the tumor. Among thyroid cancers, a great majority of differentiated thyroid caner 
(DTC) patients can be cured with the conventional treatment regimen composed of surgery, radioactive iodine and thyroid hormone therapy. Less than $10 \%$ of DTC patients present with or ultimately develop distant metastases (Lee and Soh., 2010) .'In radioactive iodine resistant subjects, conventional chemotherapy response rates are low and long term cure is rare, for patients with medullary thyroid cancer (MTC), which comprises around 5\% of thyroid cancers, the only curable treatment choice is surgery. For the treatment of anaplastic thyroid caner (ATC), which is luckily the least common histologic type of thyroid cancer $(\sim 1 \%)$, surgery, radiotherapy and chemotherapy have been used with disappointing results.. (Sherman, 2010).

\section{MATERIALS AND METHOD}

A case - control study approach a quantitative design was carried out. The subjects of the study consisted of (172) clients divided into two groups, a case group (86) clients with thyroid cancer and a control group (86) clients without goiter and thyroid cancer. (66) case and
(9) control were from Nuclear Medicine and Oncology Hospital, (14) case and (52) control were from AL-Jamhori Teaching Hospital, (6) case and (25) control from Al-Salam Teaching Hospital was chosen according to the following criteria: their age ranges between (20-60) years. Both gender (males and females). The patients who diagnosed with thyroid cancer and visited the thyroid consultation in The Nuclear Medicine and Oncology Hospital for laboratory investigation (T3, T4, and TSH.), ultra sound, or physical department to receive radio active iodine. The patients who visited AL-Jamhori Teaching Hospital, and Al-Salam Teaching Hospital and complained of signs and symptoms of thyroid cancer according to the histology type or diagnosed with thyroid cancer and need for thyroidectomy. The exclusion criteria of the sample in the study were: They were free from any disease related with goiter and thyroid cancer. Their age was between 20 years and 60 years . They agreed to participate in the study.

\section{RESULTS}

Table (1): Socio-Demographic Characteristic of the Study and control sample (No. 172).




Table (2): Correlation between study and control groups for the risk factors of thyroid cancer

\begin{tabular}{|c|c|c|c|c|c|c|c|c|}
\hline \multirow{2}{*}{\multicolumn{2}{|c|}{ Items }} & No. & $\%$ & No. & $\%$ & \multirow{2}{*}{$\mathrm{X}^{2} \mathrm{cal}$} & \multirow{2}{*}{$\mathrm{X}^{2}$ tab. } & \multirow{2}{*}{ D.F } \\
\hline & & \multicolumn{2}{|c|}{ Case } & \multicolumn{2}{|c|}{ Control } & & & \\
\hline \multirow{2}{*}{$\begin{array}{l}\text { Family had previous } \\
\text { history for goiter }\end{array}$} & Yes & 50 & 58.1 & 12 & 14.0 & \multirow{2}{*}{36.418} & \multirow{2}{*}{$\begin{array}{c}3.84 \\
(0.05)\end{array}$} & \\
\hline & No & 36 & 41.9 & 74 & 86.0 & & & 1 \\
\hline \multirow{2}{*}{$\begin{array}{l}\text { positive family history } \\
\text { for thyroid cancer }\end{array}$} & Yes & 19 & 22.1 & 86 & 100.0 & \multirow[b]{2}{*}{109.752} & \multirow{2}{*}{$\begin{array}{c}3.84 \\
(0.05)\end{array}$} & 1 \\
\hline & No & 67 & 77.9 & 0 & 00.0 & & & \\
\hline \multirow{4}{*}{$\begin{array}{l}\text { Exposed to radio active } \\
\text { iodine }\end{array}$} & Never & 30 & 34.9 & 86 & 100.0 & \multirow{4}{*}{83.034} & \multirow{4}{*}{$\begin{array}{c}7.82 \\
(0.05)\end{array}$} & \\
\hline & Once & 36 & 41.9 & 0 & 00.0 & & & 3 \\
\hline & Twice & 17 & 19.8 & 0 & 00.0 & & & \\
\hline & $\begin{array}{l}\text { Third or } \\
\text { more }\end{array}$ & 3 & 3.5 & 0 & 00.0 & & & \\
\hline \multirow{2}{*}{ Smoking status } & Yes & 11 & 12.8 & 5 & 5.8 & \multirow{2}{*}{2.481} & \multirow{2}{*}{$\begin{array}{c}3.84 \\
(0.05)\end{array}$} & 1 \\
\hline & No & 75 & 87.2 & 81 & 94.2 & & & \\
\hline \multirow{4}{*}{$\begin{array}{l}\text { Head and neck x-ray } \\
\text { before cancer } \\
\text { diagnosing }\end{array}$} & None & 45 & 52.3 & 58 & 67.4 & \multirow{4}{*}{6.424} & \multirow{4}{*}{$\begin{array}{c}7.82 \\
(0.05)\end{array}$} & \multirow{4}{*}{3} \\
\hline & Once & 19 & 22.1 & 9 & 10.5 & & & \\
\hline & Twice & 6 & 7.0 & 8 & 9.3 & & & \\
\hline & Third & 16 & 18.6 & 11 & 12.8 & & & \\
\hline
\end{tabular}

p Value $\leq$ 0.05.

\section{DISCUSSION}

\section{Age groups}

The analysis of the results with regard to demographic characteristics of study group and control group indicate that the age group of (5059 years) constitutes the highest percentage $(34.0 \%)$ of the total sample (Table 1). Although the reason for the association between age and outcome is not entirely clear, thyroid cancer can occur in all ages but it does refer to the fact that incidence of thyroid cancer may increase in middle or older age according to the results because most people who develop thyroid cancer in the sample were of this age group.

Our finding disagrees with Moazezi Zoleika and others (2011) in Iran who indicated that the patients age group was between (35-55 years) at (45\%), While Iftikhar and others (2011) in Pakistan found that the most affected age group with thyroid cancer was 31-40 years with $(27.6 \%)$.

\section{Gender}

The present study shows that the highest level of the present study was female and constituted $(78.0 \%)$ (Table 1). The pronounced gender disparity suggests that hormones play an important role in the etiology of thyroid cancer because thyroid carcinoma cells have both estrogen Alfa and estrogen Beta receptors. Estrogen receptors are widely expressed in neoplastic thyroid tissue and there is evidence that estrogen may contribute to thyroid carcinogenesis (Rajoria and et al, 2010).
The present study is in agreement with Rahmani and Raisian (2013) in Iran, who found that the female gender constituted $33(82.5 \%)$ and seven (17.5\%) males. While Qari (2004) in her study of Pattern of thyroid malignancy at a university hospital in western Saudi Arabia. stated that $(53.3 \%)$ were 24 males and $(46.7 \%)$ were 21 females

\section{Residence}

The result of the present study indicates that $(63.0 \%)$ of the study group and $(78.0 \%)$ of control group were living in urban areas (Table 1). This could potentially confound interpretation of the urban-rural gradients such that the risk may not be entirely related to urban or rural factors, but rather to the economic climate of the areas. Urban participants may have higher expectations of cancer care and felt that the cancer care they received fell below expectations.

The result of the present study is similar to those by Zivaljevic and others (2004) in Serbia who found that urban patients constituted $(68.2 \%)$ and the rural patients constituted $(31.8 \%)$ of study group.

\section{Marital status}

The result of the present study indicates that the majority of the patients with thyroid cancer were married and accounted for $(88.0 \%)$ and $(81.0 \%)$ of the control group (Table 1$)$. The individuals that participated in this study were between (20-60) and in this age most people in our community are married.

Zivaljevic and others (2004) support our finding as they found that $(75.4 \%)$ was married. 
Monica María and others (2010) found that the majority of the case group were married and constituted $(50 \%)$.

\section{Occupation and thyroid cancer}

The result of the present study indicates that $(72.0 \%)$ of thyroid cancer patients were housewives, also $(63.0 \%)$ of the control group were housewives (Table 1). Occupations were not thought to be associated with an increased risk for thyroid cancer. The role of specific occupations in any one individual's diagnosis of thyroid cancer could not be reliably evaluated. But the result of the present study is interpreted that the nature of the sample included a high percentage of females more than male and most of them were housewives.

The current study is in agreement with Moazezi and others (2011) who found that (69.6\%) patients diagnosed with thyroid cancer were housewives, while it differs from Zivaljevic and others (2004) who found that (35.4\%) patients diagnosed with thyroid cancer were retired.

\section{Educational level and thyroid cancer}

The result of the present study indicates that the high percentage of both study and control group were in illiterate, $(28.0 \%)$ for case group and $(31.0 \%)$ for control group (Table 1).

This disagrees with Therese and Laurent (2005) who found that (51.5\%) among their patients were able to read and write and (7.5\%) never went to school. Also Zivaljevic and others (2004) found that $(55.4 \%)$ among case group were Primary school graduates.

\section{Concomitant with chronic diseases}

The result of the present study indicates that a high percentage of patients were never concomitant with chronic diseases among case and control group, $(71.0 \%)$ for case and $(80.0 \%)$ for control group (Table 1). There is a lack of an overall association between chronic diseases and thyroid cancer and the association with most chronic diseases depended upon cancer type, gender, and age.

\section{Risk factors of thyroid cancer}

\section{Previous family history for goiter}

The study findings revealed that there was a significant statistical association of previous family history with goiter and thyroid cancer patients at $(p<0.05)($ Table 2$)$. This indicates that individuals may suffer from thyroid cancer from his family. Most of the patients interviewed had previous mother and sister's history of goiter and some of them had previous father history for goiter. Therefore, previous family history for goiter is considered the most common risk factors for thyroid cancer according to the results.

The result of the present study is in agreement with Juliane and others (2008) who indicated that about $(50.5 \%)$ of patients with thyroid cancer showed a positive family history for goiter. In contrast, only $25 \%$ of patients with normal thyroids had a positive family history for goiter $(\mathrm{P}<0.001)$. Patients with goiters had a significantly higher proportion of parents $(\mathrm{P}<0.001)$ or siblings $(\mathrm{P}=0.004)$ with goiters.

\section{Positive family history for thyroid cancer}

The result of the present study indicates that there is a significant statistical association of positive family history for thyroid cancer at ( $p$ $<0.05$ ) (Table 2). Apparent familial thyroid cancer development has been reported by several clinicians, including cases which seem to show a dominant pattern of inheritance. Thyroid carcinomas occur rarely as part of several familial syndromes, which may involve hereditable loss of tumor suppressor genes. The familial risk ratio for individuals with a family history of PTC ranges from 8.6- to 10.3-fold of increased risk of developing a thyroid tumor, suggesting an inherited genetic influence on the development of the disease (Marco and et al, 2008).

The result of the present study is similar to Haggi and others (2012) who found that thirtyseven $(10 \%)$ patients had a family history of thyroid cancer; all of which had papillary thyroid cancer. While Qari (2004) found that there was no family history of thyroid cancer in her patients.

\section{Exposed to radio active iodine}

The result of the present study indicates that there is a significant statistical association of exposure to radio active iodine and thyroid cancer at ( $\mathrm{p}$ value < 0.05) (Table 2). Most patients are treated with a dose of radioactive iodine that will destroy the overactive cells in the nodule. Data analysis shows that there are (36) patients in the study who were exposed to radio active iodine one time only after they were diagnosed with thyroid cancer, (17) patients were also exposed to radio active iodine two times, (5) of them had previous history of thyrotoxicosis (hyperthyroidism) before cancer diagnosing and others were exposed to radio active iodine after diagnosing thyroid cancer, and (3) patients were exposed to radio active iodine 3 times or more. All of them had a history of toxic goiter. This explains that if the individual had a history of toxic goiter and was exposed to radio active iodine thyroid scan test should be done to 
measure the amount of radiation in the gland. Radio active iodine is a capsule take orally and it may be given to the patient as single dose then the patient will isolate 48 hours after ingesting the capsule or it may be given as two dose, the second dose will be take after 24 hours of first dose, the third dose will take after week of second dose.

Thyroid cancer patients also have further testing, whole-body radioiodine scans using a "tracer" dose of RAI. If their results are not "clean," they may then receive treatment with another dose of RAI in an attempt to eliminate remaining thyroid cells. Abnormal areas of the thyroid that have less radioactivity than the surrounding tissue are called cold nodules, and areas that take up more radiation are called hot nodules. Hot nodules are usually not cancerous, but cold nodules can be benign or cancerous. Because both benign and cancerous nodules can appear cold, this test by itself can not diagnose thyroid cancer. The present study shows that if the patient exposed to radio active iodine two time or more and thyroid scan test was cold, he may have thyroid cancer and the increasing exposure times to radio active iodine affect the result of thyroid scan test and cause thyroid cancer (Leah, 2010).

The result of the present study is in agreement with Sawka and others (2009) who found that the risk of second primary malignancies in thyroid cancer survivors treated with RAI was significantly increased at $(\mathrm{p}=$ 0.010).

\section{Smoking status}

The result of the present study shows that there is no significant relationship between smoking status and thyroid cancer at ( $\mathrm{p}$ value $<$ 0.05 ) (Table 2). The finding of the present study accounted for only 11 patients who were smoker in the case group and 5 clients who were in the control group. This number of smokers may not affect the result of the study because there is no noticeable difference between the two groups and there is a small number of smokers in the whole sample. Also most individuals in the sample were females.

Our finding is similar to that by Wendy and others (2002) who found that smoking was not associated with thyroid cancer risk. Also Elzbieta and others (2011) demonstrated that patients with thyroid cancer smoked cigarettes less frequently than subjects in the control group (29.93\%, 43.10\%, respectively). This was not considered a risk for thyroid cancer or protective factors (at $\mathrm{p}=0.003)$.

\section{Head and neck x-ray before diagnosing thyroid cancer}

The result of the present study shows that there is no significant statistical difference of exposure to radiation for head and neck and thyroid cancer at $(\mathrm{p}$ value $<0.05)$ (Table 2 ). Exposure to radiation in both cases and controls may have been underreported, because much of the exposure would have occurred in infancy or childhood and would not be remembered. Also, history of thyroid problems may have been reported inaccurately by cases. Previous exposure to head and neck irradiation in childhood, adolescence or even adulthood may be an important contributing factor for the development of thyroid cancer but this depends on the age of the individual and numbers of x-ray exposure. It has become recognized that the thyroid gland is particularly sensitive to the effects of radiation resulting in thyroid tumors with a risk for cancer. In addition, patients who require radiation for the treatment of certain types of cancer in or near the head and neck area may also have an increased risk for the development of thyroid nodules and thyroid cancer, but in the present study there are no cases was had other types of cancer except thyroid cancer.

The result of the present study disagrees with that by Memon and others (2010) in Kuwait who found that there was approximately a twofold increased risk for thyroid cancer among individuals who were exposed to dental $\mathrm{x}$-rays at $(\mathrm{P}=0.001)$.

\section{CONCLUSIONS}

There is real variation in age group, since middle age and elderly have more risk for thyroid cancer than others. Also, the incidence of thyroid cancer is reported to be of high percentage among females compared to males. Thyroid cancer has been significantly associated with previous family history for goiter, positive family history for thyroid cancer and exposure to radio active iodine, but not with smoking and x-ray for head and neck before cancer diagnosing. Salt water and canned tuna fish (a source of iodine) were significantly associated with thyroid cancer and could be important protective factors from thyroid cancer. The correlation between age group and type of thyroid cancer shows that middle age and elderly had papillary thyroid carcinoma in high percentages higher than the other age groups. The incidence of differentiated thyroid carcinoma, especially papillary thyroid carcinoma according to histopathology was more among women compared with men. Thyroid 
inhibiter drugs used for toxic goiter before thyroid cancer diagnosing had significant relationship with thyroid cancer among the case study.

\section{RECOMMENDATIONS}

An educational program is recommended about the prevention of thyroid cancer through information about the risk of exposure to radiation and avoidance of goitrogens, substances that block thyroid hormone production which can cause goiters and thyroid cancer and encourage some types of diet which are sources of iodine, so using salt iodine by explaining the daily requirement of body and thyroid gland for iodine and, a thyroid supporting protocol would be recommended in collaboration with Health Governorate, medical colleague, nursing colleague and the World Health Organization. Providing specialists in nuclear medicine in the Consultation of Oncology and Nuclear Medicine Hospital or establishment of another center in Mosul city for screening, monitoring, treatment and nursing management for thyroid cancer. Supplying T3, T4 and TSH tests in all hospitals which carry out these tests and providing the Oncology and Nuclear Medicine Hospital with radioactive iodine doses because sometimes it is not available and most patients diagnosed with differentiated thyroid cancer and toxic goiter are in bad need of this treatment. Proceeding future researches on the prevalence of thyroid cancer effects of risk factors and dietary habits on thyroid gland in Mosul city.

\section{REFERENCES}

National Cancer Institute (NCI). (2012). Surveillance Epidemiology and End Results. Stat Facts Sheet(SEER) http://seer.cancer.gov/statfacts/html/thyro. $\underline{\mathrm{html}}$

Harrison's, A. (2004). Principles of Internal Medicine. $\left(16^{\text {th }}\right.$ ed.).

McGraw-Hill Professional-.3. P.p. 99.

Marjani, A.; Kabir, M.J. (2008). Incidence of thyroid cancer in Golestan Province of Iran: Some initial observations. Pak J Med Sci. 24(6). P.p. 887-90

Sherman, S.I. (2003). Thyroid carcinoma . The Lancet .; 361. P.p. 501-512.

Boice, J.D. (2005). Radiation-induced Thyroid Cancer - What's New?. J Natl Cancer Inst 97. P.p. 703-5.

Barbra, L. Christensen and Elaine, O. Kockrow. (2011). Adult health nursing . $\left(16^{\text {th }}\right.$ edition). By Mosby, Inc. an affiliate of Elsevier Inc. P.p.501.

Hegedus, L. (2004). Clinical practice, The thyroid nodule. $N$ Engl J. Med . 351. P.p. 1764-71.

Lee, J.; Soh, E.Y. (2010). Differentiated thyroid carcinoma presenting with distant metastasis at initial diagnosis clinical outcomes and prognostic factors. Ann Surg. 251. P.p.114-119.

Sherman, S.I. (2010). Cytotoxic chemotherapy for differentiated thyroid carcinoma. Clin Oncol (R Coll Radiol). 22. P.p.464-468.

Moazezi, Zoleika; Mahmoudi, Mahmoud; AliReza, Alaleh; Yahyahpour, Y. (2011). risk factors of thyroid cancer in Babol, Northern Iran . Casp J Intern Med. 2(1). P.p. 171-177.

Iftikhar, A.; Khush, A.; Khwaja A.; Mati, H.; , Karim, K. and Hameed, N. (2011). Patterns of Differentiated Thyroid Cancer in Baluchistan Province of Pakistan: Some Initial Observations . Med J Malaysia. 66 ( 4). P.p. 322-325.

Rajoria, S; Suriano, R; Shanmugam, A; Wilson, Y.L.; Schantz, S.P.; Geliebter, J.; Tiwari, R.K.; (2010). Metastatic phenol- type is regulated by estrogen in thyroid cells. Thyroid. (20). P.p.33-41.

Rahmani, Nasrin; and Raisian, Mohammad. (2013). Clinical management and outcomes of papillary, follicular and medullary thyroid cancer surgery . Medicinski Glasnik, Sari, Iran . 10(1). P.p. 164.

Qari, Faiza. (2004). Pattern of thyroid malignancy at a university hospital in western Saudi Arabia. Saudi Med J . 25 (7). P.p.447.

Zivaljevic, Vladan,; Vlajinac, Hristina,; and Jankovic, Radovan. (2004). Case-control study of anaplastic thyroid cancer. Tumori. 90. P.p. 9-12.

Monica, María,; Rochy, M.; Shirley, Alexandra; Yady, Karina; and Maryori, Pertuz. (2010). Psychological well-being and quality of life in patients treated for thyroid cancer after surgery. terapia psicologica. 28(1). P.p. 69-84.

Therese, Truong,; Laurent, Orsi,; and et al. (2005) . Role of Goiter and of Menstrual and Reproductive Factors in Thyroid Cancer: A Population-based Case-Control Study in New Caledonia (South Pacific), a Very High Incidence Area. $\mathrm{Am} J$ Epidemiol .161. p. p.1056-1065. 
Juliane, Wicht,; Joerg, Singer, and Ralf, Paschke. (2008). Genetic predisposition for goiters analysed by a case-control study. Endocrine Abstracts. 16. P.p.715.

Marco, Capezzone; Silvia, Cantara; Stefania, Marchisotta; Sebastiano, Filetti; Maria, Margherita De Santi; Benedetta, Rossi; Giuseppe, Ronga; Cosimo, Durante; and Furio Pacini. (2008). Short telomeres, hTERT gene amplification and increased telomerase activity in the blood of familial papillary thyroid cancer patients. J. Clin Endocrinol Metab. 93. P.p.3950-7.

Haggi, Mazeh,; Joy, Benavidez; Jennifer, L.; Poehls, Linda, Youngwirth,; Herbert, Chen,; and Rebecca, S. (2012). In patients with thyroid cancer of follicular cell origin, a family history of nonmedullary thyroid cancer in one first degree relative is associated with more aggressive disease. Thyroid Academic Journal. 22(1). P.p.3-8.

Leah, Guljord; Low-Iodine Cookbook. ThyCa. (2010). Thyroid Cancer Survivors' Association, Inc.SM. ( $7^{\text {th }}$ ed.). P.p.11.

Sawka, Am,; Thabane, L.; Parlea, L.; IbrahimZada, I.; Tsang, R.W.; Brierley, J.D.; Straus, S.; Ezzat, S.; and Goldstein, D.P. (2009). Second primary malignancy risk after radioactive iodine treatment for thyroid cancer: a systematic review and meta-analysis. Thyroid. (19)5. P.p. 451507.

Wendy, J. Mack ; Susan, Preston-Martin ; Leslie, B.; and Dajun, Q. (2002). Lifestyle and Other Risk Factors for Thyroid Cancer in Los Angeles County Females. Mosby Elsevier . 12(6). P.p.395-401.

Elzbieta, Bandurska-Stankiewicz; Ewa, Aksamit-Białoszewska,; Joanna Rutkowska; Aleksander, Stankiewicz; and Danuta, Shafie. (2011). The effect of nutritional habits and addictions on the incidence of thyroid carcinoma in the Olsztyn province of Poland. Polish Journal of Endocrinology Tom. 62. (2). P.p.145150.

Memon, Anjum; Godward, Sara; Williams, Dillwyn; Siddique, Iqbal and AL-salehand, Khalid. (2010). Dental x-rays and the risk of thyroid cancer: A case-control study . Acta Oncologica article. 49. P.p.447-453. 